\title{
Hypervitaminia B12: An Unknown Endocrine Marker
}

\author{
Zulfiqar A. A. ${ }^{1}$, Sebaux A. ${ }^{1}$, Andrès E. ${ }^{2}$, Novella J. L. ${ }^{1}$ \\ ${ }^{1}$ Department of Internal Medicine and Geriatrics, University Hospital of Reims, Reims, France \\ ${ }^{2}$ Department of Internal Medicine, University of Strasbourg, Strasbourg, France
}

Email address:

abzulfiqar@laposte.net (Zulfiqar A. A.), aazulfiqar@chu-reims.fr (Zulfiqar A. A.)

\section{To cite this article:}

Zulfiqar A. A., Sebaux A., Andrès E., Novella J. L.. Hypervitaminia B12: An Unknown Endocrine Marker. Journal of Food and Nutrition Sciences. Vol. 3, No. 2, 2015, pp. 32-34. doi: 10.11648/j.jfns.20150302.11

\begin{abstract}
Measurement of serum cobalamin (vitamin B12) is routinely used in Endocrinology/Metabolism in order to diagnose or rule out a suspected Cobalamin deficiency. Hypervitaminemia B12 or high serum level of cobalamin B12 is a clinical underestimated abnormality. A high fraction of patients have high cobalamin levels and no consensus exists regarding the clinical implications for this endocrine marker, which can be involved in serious medical situations like solid neoplasms, hepatopathy, hemopathy, renal failure, and also in case of functional deficiency. We describe four clinical cases illustrating this problem, and possible links between Hypervitaminia B12 and malignant diseases, potentially engaging the prognosis.
\end{abstract}

Keywords: Hypervitaminia B12, Solid Neoplasms, Hemopathy, Renal Failure, Hepatic Disorders

\section{Introduction}

In clinical practice, measurement of serum cobalamin is requested widely for the biochemical assessment of cobalamin deficiency (1). Daily intake of 2 to $5 \mu \mathrm{g}$, together with efficient absorption, transportation, and transformation, are needed to maintain health.

Hypervitaminia B12 is a biological abnormality which remains unknown and underestimated in clinical practice. Serious pathological entities are associated. In the literature, there are few studies or case reports describing this entity, but there is no clear consensus about this endocrine marker for physicians. In addition, there are no clear prospective studies describing the effect and the meaning of hypervitaminia B12. We report four clinical cases illustrating this problem.

\section{Case History}

Case History 1: A patient of 76 years consulted for asthenia. She had a medical history including a nonautoimmune hypothyroidism treated and anxiety-depressive syndrome also treated. Clinical examination had no specific abnormalities. No weight loss was noted. The body mass index was at $23 \mathrm{~kg} / \mathrm{m} 2$. No lymphadenopathy and no suspicious mass were found. No gynecological or gastrointestinal blood loss was found for the patient. The neurological and joint articular examination was normal. Cognitive assessment found no abnormalities. We performed a full biological assessment which finds a hypervitaminia B12 estimated at $1,103 \mathrm{pg} / \mathrm{ml}$ (normal rate between 191 and $663 \mathrm{pg} / \mathrm{ml}$, in our Laboratory, by electroluminescence). This endocrine marker was therefore increased $(>663 \mathrm{pg} / \mathrm{ml})$. No deficiency or excess of vitamin folate was detected. No hepatic disturbance, no renal failure, and no anemia were found. Biological markers of malnutrition such as albumin and prealbumin were normal. No lymphopenia was observed for this patient. Thyroid function was normal. No biological inflammatory syndrome (CRP, erythrocyte sedimentation rate, fibrinogen) was found. The electrophoresis of serum did not show any abnormalities in favor of hemopathy. A homocysteine was prescribed in order to find a functional deficiency of vitamin B12, proving to be normal. No excess intake of vitamin B12 was found. We prescribed a thoracoabdomino-pelvic scanner in order not to avoid a solid neoplasia which may explain this hypervitaminia B12. A large mass of solid shape, $86 \mathrm{~mm}$ in diameter, growing at the expense of the myometrium was found; this confirmed by endo-vaginal ultrasound, with intratumoral vascular, corresponding to an uterine fibroid. The ovaries were normal. Gynecological support is recommended. 
Case History 2: A patient of 91 years, male, was hospitalized for a puncture evacuative ascites associated with terminal chronic heart failure, in polyvascular patient (pacemaker, heart rhythm disorders ...). We conducted investigations on this ascites with bacteriological and anatomo-pathological tests, which were negative. Laboratory tests at its entrance had detected a hypervitaminia B12, estimated at more than $800 \mathrm{pg} / \mathrm{ml}$ (normal rate between 191$663 \mathrm{pg} / \mathrm{ml}$ in our Laboratory, by electroluminescence). So we asked for additional tests, including a thoraco-abdominopelvic CT scan that showed cardiomegaly and ascites, with no other abnormalities such as neoplasia. No biological hepatic abnormalities and no biological inflammatory syndrome were detected; the serum protein electrophoresis was normal. On the other side, we noted severe renal impairment (creatinine clearance estimated at $29 \mathrm{ml} / \mathrm{min}$ MDRD), which explains the occurrence of hypervitaminia B12. Therapeutic adaptation was performed.

Case History 3: A patient of 92 years, female, with no notable medical history, was admitted for a deterioration of general condition, with anorexia and asthenia. On physical examination, she presented only mucocutaneous pallor. Biologically, the patient initially presented a cholestasis without jaundice character, and without cytolysis. Moreover, a hypervitaminia B12 was found, estimated at $1,469 \mathrm{pg} / \mathrm{ml}$ (normal rate between 191-663 pg/ml). We did not find abnormalities of renal and thyroid function, and a moderate biological inflammatory syndrome. Liver ultrasound was performed, finding a dilatation of the intrahepatic bile ducts and also a dilatation of the principle bile duct with no visible obstacle. We noted worsening liver function tests, with the appearance of a hepatic cytolysis, and worsening of a cholestatic jaundice. Alongside the worsening of liver function tests, there was an increase of the hypervitaminia $\mathrm{B} 12$, estimated at 1,642 $\mathrm{pg} / \mathrm{ml}$ (normal rate 191-663 pg/ml). An abdomino-pelvic CT scan had found dilated intrahepatic and extra hepatic bile ducts, upstream of a stenosis of the common bile duct, with an undercover aspect of the hilum. Stenosis of the portal vein was also found. A probable tumor process at the extra hepatic bile duct was diagnosed. Unfortunately, the patient died a few days later.

Case History 4: A patient of 89 years, female, with no notable history, was admitted for a fall without loss of consciousness, accompanied by dizziness. For several weeks, she had a poor general condition. Physical examination found only amyotrophy of members; no peripheral lymphadenopathy was palpated. Biologically, we did not find anemia, no abnormalities of renal, thyroid and liver functions. We did not note biological inflammatory syndrome, and serum albumin was at $38 \mathrm{~g} / \mathrm{l}$ (normal rate $>35 \mathrm{~g} / \mathrm{l}$ ). However, Hypervitaminia B12 was found, estimated to $1,520 \mathrm{pg} / \mathrm{ml}$ (normal rate between 191-663 pg/ml). Because of this poor condition and discovery of this high hypervitaminia B12, we decided to carry out further investigations. A thoracoabdomino-pelvic scanner had been realized. This CT scan revealed a malignant tumor of the urothelium of the left pelvis and lower caliceal group of the left kidney, responsible for peri-aortic lymph nodes, but also liver, bone, brain, cerebellar and bulbar metastases. Cancer with significant metastasis was diagnosed. An announcement of the diagnosis was made to the patient. After a multidisciplinary meeting and due to a poor prognosis, palliative care had been decided.

\section{Discussion}

The association between elevated plasma cobalamin levels and cancer risk is poorly known. A high prevalence of elevated cobalamin levels has been reported in patients with liver cancer (2), other solid neoplasms (3), and hematological malignancies (4). Some studies have indicated a high prevalence of cancer, both hematological and solid tumors, among patients with high cobalamin levels (5) Elevated plasma cobalamin levels have also been associated with several non-malignant diseases, including liver diseases, alcoholism and alcoholic cirrhosis (decrease in hepatic tissue uptake and cellular vitamin B12 and complex haptocorrincobalamin are the main mechanisms implicated (4)), and renal failure, autoimmune and infectious diseases (6). The role of the kidney in the metabolism of vitamin B12 is now clear, but not completely understood. Kidney failure is one of the reasons to search; this mainly linked by an accumulation of serum transcobalamins. Hypervitaminia B12 can be observed in case of functional deficiency with the need to dose homocysteine (4).

Clinically, it can be sometimes paradoxically accompanied by signs of deficiency reflecting a functional deficit in relation to qualitative abnormalities related to defects in tissue uptake and action of vitamin B12.

The major cancers implicated are hepatocellular carcinoma and secondary liver tumors, breast cancer, colon cancer, stomach cancer and pancreatic tumors (4). In liver tumors, the main mechanisms implicated in the genesis of hypervitaminia B12 are decreased hepatic clearance of complex haptocorrin-cobalamin and excess degradation of hepatocytes (4). Hypervitaminia B12 is frequently observed in malignant hematological abnormalities. It is basically linked to myeloproliferative disorders, including chronic myelomonocytic leukemias and primary hypereosinophilic syndrome, myelodysplastic syndromes and acute leukemias, including promyelocytic leukemias (4). This is mainly due to granulocyte production of haptocorrin.

A recent study showed that levels of the circulating cobalamin binding protein haptocorrin were high in patients with high plasma cobalamin levels (5). Moreover, cancer was associated with high cobalamin and high haptocorrin levels (7). This protein originates from a variety of tissues, but its physiological function remains unknown (8). It is elevated in patients with some cancer types (9) and has been suggested as a marker for disease progression (9). From a point of view prognostic, the correlation found in some cases between the size of certain tumors, including liver, and the degree of elevation of the vitamin B12 was suggested for hypervitaminia $\mathrm{B} 12$ as a possible tumor marker of poor prognosis (10). Geissbühler et al attributed to the vitamin 
$\mathrm{B} 12$, a prognostic value in a tumor disease (11). The vitamin $\mathrm{B}_{12} /$ C-reactive protein Index $(\mathrm{BCI})$ has been proposed as a prognostic indicator in patients with advanced cancer (11). Geissbühler et al emphasize the relevance of vitamin B12 levels in patients with cancer: continuing or stopping a curative treatment, pursuit or stop further investigations (11).

Etiological profile of hypervitaminemia B12 has mostly serious diseases entities and for which early diagnosis is crucial to the plan rather than prognostic. These entities are represented mainly by solid malignancies, hematological malignancies and liver diseases.

This reflects the potential significance that may have the dosage of vitamin B12 as an early marker of diagnosis of these diseases.

\section{Conclusions}

Codified approach is needed to determine the potential indications of the search for a hypervitaminemia B12 and practice what to do before the discovery of a high serum level of cobalamin. Finding a high plasma concentration of vitamin B12 should lead to a systematic search for a tumor or a hepatic disease. Referent laboratory should actively advertise the numerous diseases implicated with high level of vitamin B12. A prospective study is underway in acute geriatric unit to investigate the prevalence and causes explaining hypervitaminia B12. A systematic search of these conditions should be performed including in geriatrics, in order not to delay an appropriate treatment. Hypervitaminia $\mathrm{B} 12$, in geriatrics, could be a real marker in the diagnosis and prognosis orientation of these conditions.

\section{References}

[1] Carmel R. Biomarkers of cobalamin (vitamin B-12) status in the epidemiologic setting: a critical overview of context, applications, and performance characteristics of cobalamin, methylmalonic acid, and holotranscobalamin II. Am J Clin Nutr. 2011; 94(1):348S-358S.
[2] Norredam K, Chainuvati T, Gimsing P, Hippe E, Viranuvatti V. Plasma cobalamin and transcobalamin in patients with primary carcinoma of the liver. A study from Thailand. Scand J Gastroenterol. 1983; 18(2):229-232.

[3] Chiche L, Jean R, Romain F, et al. Clinical implications of high cobalamin blood levels for internal medicine. Rev Med Intern. 2008; 29(3):187-194.

[4] Serraj K, Mecili M, Housni I, Andrès E. Hypervitaminemia B12 (high level of cobalamin): physiopathology, role and interest in clinical practice. Presse Med. 2011;40(8):1120 1127.

[5] Arendt JF, Nexo E. Cobalamin related parameters and disease patterns in patient's with increased serum cobalamin levels. PLoS One. 2012; 7(9):e45979.

[6] Arendt JF, Nexo E. Unexpected high plasma cobalamin: proposal for a diagnostic strategy. Clin Chem Lab Med. 2013; 51(3):489-496.

[7] Johan Frederik Berg Arendt, Lars Pedersen, Ebba Nexo, Henrik Toft Sorensen. Elevated plasma Vitamin B12 Levels as a Marker for Cancer: A Population-Based Cohort Study. JNCI 2013, Vol. 105, Issue 23 | December 4.

[8] Morkbak AL, Poulsen SS, Nexo E. Haptocorrin in humans. Clin Chem Lab Med. 2007; 45(12):1751-1759.

[9] Zittoun J, Zittoun R, Marquet J, Sultan C. The three transcobalamin in myeloproliferative disorders and acute leukaemia. Br J Haematol. 1975; 31(3):287- 298.

[10] Wheeler K, Pritchard J, Luck W, Rossiter M.Transcobalamin I as a marker for fibrolamellar hepatoma. Med Pediatr Oncol. 1986; 14(2): 227-9.

[11] Geissbühler P, Mermillod B, Rapin C-H. Elevated Serum Vitamin B12 Levels Associated With CRP as a Predictive Factor of Mortality in Palliative Care Cancer Patients: A Prospective Study Over Five Years. J Pain Symptom Manage. 2000; 20(2):93-103. 